\title{
Relación entre los puntajes del índice de pendiente del segmento ST/frecuencia cardiaca y el índice del cambio del segmento ST sobre el cambio de la frecuencia cardiaca durante la realización de la prueba de esfuerzo convencional con la presentación de nuevos eventos cardiovasculares
}

\author{
Ángel Alberto García-Peña ${ }^{a, *}$, Ana M. Barón ${ }^{a}$, Edna Salazar ${ }^{a}$, Óscar M. Múñoz ${ }^{a}$, \\ María Rueda ${ }^{\text {b e Iván González }}{ }^{\text {b }}$ \\ a Medicina Interna y Cardiología, Unidad de Cardiología, Hospital Universitario San Ignacio, Pontificia Universidad Javeriana, \\ Bogotá, Colombia \\ ${ }^{\mathrm{b}}$ Facultad de Medicina, Pontificia Universidad Javeriana, Bogotá, Colombia
}

Recibido el 15 de diciembre de 2015; aceptado el 4 de septiembre de 2016

Disponible en Internet el 27 de octubre de 2016

\author{
PALABRAS CLAVE \\ Test; \\ Ejercicio; \\ Epidemiología; \\ Episodio \\ cardiovascular
}

\begin{abstract}
Resumen
Introducción: La prueba de esfuerzo convencional es una herramienta diagnóstica de fácil interpretación y accesibilidad que se utiliza de forma frecuente en la evaluación del dolor torácico, no obstante, sus características operativas están influenciadas por múltiples variables resultando en una capacidad limitada para la predicción global de desenlaces cardiovasculares. El uso de los índices y las variables adicionales en su interpretación podrían mejorar la predicción de los desenlaces cardiovasculares.

Métodos: Mediante la realización de un estudio descriptivo, analítico y retrospectivo, se evaluó la relación y capacidad de predicción de los índices pendiente ST/FC y $\triangle \mathrm{ST} / \Delta \mathrm{FC}$ con el desarrollo de eventos cardiovasculares a un año. De un total de 438 estudios, se seleccionaron 138 que cumplían con los criterios para evaluación. Se realizó un análisis descriptivo de las variables de interés y posteriormente se procedió a realizar un análisis univariado y multivariado de las diferentes variables y desenlaces de interés.

Conclusiones: Se encontró una relación estadísticamente significativa entre la capacidad del ejercicio, el índice pendiente ST/FC y el desarrollo de los desenlaces cardiovasculares, sin encontrar relación significativa con el índice pendiente $\Delta S T / \Delta F C$. Se observa discreta mejoría
\end{abstract}

\footnotetext{
* Autor para correspondencia.

Correo electrónico: angel.garcia@javeriana.edu.co (A.A. García-Peña).
} 
de la capacidad de predicción global de la prueba de esfuerzo convencional con la adición del índice pendiente ST/FC (AUC 0,70 a AUC 0,74).

Relationship of ST segment/heart rate slope index and ST segment change index scores on the heart rate change during conventional stress test with the presentation of new cardiovascular events.

(C) 2016 Sociedad Colombiana de Cardiología y Cirugía Cardiovascular. Publicado por Elsevier España, S.L.U. Este es un artículo Open Access bajo la licencia CC BY-NC-ND (http:// creativecommons.org/licenses/by-nc-nd/4.0/).

\section{KEYWORDS}

Test;

Exercise;

Epidemiology;

Cardiovascular

episode

\section{Introducción}

La enfermedad cardiovascular es la principal causa de la morbimortalidad en Colombia y en el mundo ${ }^{1}$, siendo la responsable de un gran número de hospitalizaciones y procedimientos diagnósticos que generan costos significativos para el sistema de salud. El diagnóstico temprano de la enfermedad coronaria es fundamental para lograr una precoz y efectiva intervención con el objetivo de reducir la aparición del infarto agudo de miocardio, la falla cardiaca y la muerte.

Uno de los métodos más utilizados en el estudio de los cuadros del dolor torácico sugestivos de enfermedad coronaria es la prueba de esfuerzo convencional, por ser un estudio de bajo costo, fácil acceso e interpretación. La principal limitación de este estudio son sus características operativas con una sensibilidad aproximada del $68 \%$ y una especificidad del $77 \%$ para el diagnóstico de la enfermedad coronaria $^{1-4}$. Buscando mejorar la capacidad diagnóstica de esta prueba se han descrito medidas que aumentan la capacidad de predicción de la enfermedad coronaria obstructiva y de la mortalidad en los hombres y en las mujeres sin generar costos adicionales. La proporción entre la pendiente del segmento ST/FC y el índice $\Delta$ ST/ $\Delta$ FC demostraron una mejor capacidad de predicción de la enfermedad coronaria en los hombres y en las mujeres, alcanzando una especificidad del $96 \%$ y un aumento de la sensibilidad de 84 a $91 \%$ en las mujeres $(p<0,010)$, sin cambio en la sensibilidad en los hombres $(89 \%)^{5-11}$. El uso de estos índices aún es controversial y no está recomendando en las guías actuales de la Asociación Americana del Corazón ${ }^{2}$. Por esta razón, se realizó la evaluación del desempeño diagnóstico de estos índices y la potencial contribución en la capacidad de predicción del desarrollo de eventos cardiovasculares a un año (muerte, angina inestable, infarto agudo del miocardio, estudio con arteriografía coronaria, revascularización percutánea o quirúrgica u hospitalización por causa cardiovascular).

\section{Materiales y métodos}

\section{Diseño del estudio}

Se realizó un estudio descriptivo, analítico y retrospectivo en los pacientes con indicación de estudio con prueba 
de esfuerzo convencional por dolor torácico y sospecha de enfermedad coronaria. El objetivo principal fue evaluar la relación entre los índices pendiente ST/FC y $\Delta \mathrm{ST} / \triangle \mathrm{FC}$, presentación de eventos cardiovasculares y determinación del valor en el cual los índices pendiente ST/FC y $\Delta \mathrm{ST} / \Delta \mathrm{FC}$ tiene un mejor desempeño diagnóstico. Como objetivos secundarios, se evaluó la relación entre el tiempo del ejercicio, la capacidad funcional y la presencia de eventos cardiovasculares.

\section{Metodología}

Se evaluaron todas las pruebas de esfuerzo realizadas en la unidad de cardiología del Hospital San Ignacio desde octubre del 2012 hasta noviembre de 2014. Se incluyeron en el análisis aquellas pruebas indicadas para el estudio del dolor en pacientes mayores de 18 años, que hubiesen alcanzado al menos el $85 \%$ de la frecuencia cardiaca máxima esperada según la fórmula (220-edad) y que tuvieran todos los datos requeridos para el estudio. Se calculó el tamaño de la muestra con un poder del $95 \%$, una precisión del $5 \%$, una diferencia del $10 \%$ para un total de 138 pacientes. Todos los estudios se realizaron en una banda sinfín General Electric ${ }^{\circledR}$ serie T2100 con posibilidad para revisión y edición de datos mediante el protocolo de Bruce y Bruce modificado. Los índices evaluados se tomaron directamente del resultado proporcionado por el equipo, los cuales se calculan de forma automática según el promedio de la pendiente del segmento ST y su frecuencia cardiaca en todas las derivaciones durante las diferentes etapas del protocolo, así como el cambio de las mismas (Delta) entre las etapas alcanzadas durante el protocolo.

Para la evaluación de los desenlaces un año posterior a la realización de la prueba de esfuerzo convencional. Se hizo una revisión de la historia clínica electrónica del Hospital San Ignacio, se realizaron llamadas telefónicas a los números consignados en la historia clínica para determinar sí el paciente presentó algún desenlace de interés para el estudio.

\section{Resultados}

Se realizó revisión de un total de 438 pruebas de esfuerzo convencionales realizadas en Hospital Universitario de San Ignacio entre los años 2012 y 2014 que cumplían los criterios de inclusión para el estudio, 300 pruebas fueron excluidas del análisis por no contener los datos completos requeridos, seleccionado un total de 138 para el análisis final acorde con el tamaño de muestra propuesto inicialmente.

Las características demográficas y la estadística descriptiva general de las pruebas incluidas para el análisis se presentan en la tabla 1.

Se lograron evaluar los desenlaces cardiovasculares según la metodología propuesta en el $98 \%$ de los pacientes, se realizó un análisis de sensibilidad con el $2 \%$ restante (analizados como sanos y muertos), sin modificación de las conclusiones del estudio. Se encontraron un total de 57 eventos durante el primer año posterior a la realización de la prueba de esfuerzo convencional, dado el bajo número de eventos se agruparon en: la muerte, los desenlaces cardiovasculares (angina inestable, infarto agudo de miocardio, estudio con
Tabla 1 Características generales

\begin{tabular}{ll}
\hline $\begin{array}{l}\text { Edad promedio (años), DS } \\
\text { Género }\end{array}$ & $\begin{array}{l}55,9, \text { DS } 12,8 \\
\text { Masculino } 48 \%, \\
\text { Femenino } 52 \%\end{array}$ \\
$\begin{array}{l}\text { Tiempo de ejercicio promedio (min), } \\
\text { DS }\end{array}$ & 10,08, DS 2,79 \\
$\begin{array}{l}\text { Capacidad de ejercicio promedio } \\
\quad \text { Mets), DS }\end{array}$ & 10,8, DS 3,21 \\
$\begin{array}{l}\text { Índice ST/FC promedio, DS } \\
\text { Índice pendiente ST/FC, DS }\end{array}$ & 1,70, DS 0,90 \\
\hline
\end{tabular}

DS: Desviación estándar; Mets: Medida de equivalentes metabólicos.

Tabla 2 Eventos cardiovasculares

\begin{tabular}{ll}
\hline Muertes & 2 eventos \\
Hospitalización & 41 eventos \\
Desenlace cardiovascular & 14 eventos \\
(Eventos: angina 7, RVM & \\
percutánea 2, RVM quirúrgica 1, \\
IAM 2, arteriografía coronaria 2) \\
Total & 57 eventos \\
\hline
\end{tabular}

RVM: Revascularización. IAM: Infarto agudo de miocardio.

arteriografía coronaria, revascularización percutánea o quirúrgica) y la hospitalización por causa cardiovascular. Ver tabla 2.

Se realizaron pruebas de normalidad para las variables cuantitativas con el test de Kolmogorov-Smirnov, encontrando que todas las variables cumplieron criterios de normalidad, por lo que se procedió a realizar el análisis univariado y el multivariado y el análisis de regresión logística para cada uno de los eventos de interés. Ver tablas 3-5.

Se encuentra que existe relación significativa entre la capacidad de ejercicio, el índice ST/FC y el desarrollo de eventos cardiovasculares, sin encontrar relaciones estadísticamente significativas entre las demás variables evaluadas y los desenlaces de interés al ser evaluadas de forma separada y de forma conjunta. Respecto a la capacidad de predicción conjunta de las variables se encontró el área bajo la curva de 0,70 para el desarrollo de eventos cardiovasculares teniendo en cuenta las variables de rutina evaluadas en la prueba de esfuerzo, encontrando un incremento del área bajo la curva a 0,74 al tener en cuenta el índice ST/FC y sin encontrar modificación al incluir el índice pendiente $(\Delta \mathrm{ST} / \Delta \mathrm{FC})$.

\section{Discusión}

La prueba de esfuerzo convencional constituye una herramienta diagnóstica de uso rutinario en múltiples centros a nivel nacional e internacional, su fácil interpretación y disponibilidad se contraponen con sus regulares características operativas, las cuales están afectadas por múltiples variables incluyendo: el género, la capacidad de ejercicio, la severidad y el numero de arterias coronarias afectadas, por lo que el desarrollo de algoritmos e índices han intentado mejorar su capacidad de predicción, los cuales por su pobre aporte y complejidad de cálculo en algunos casos no son usados rutinariamente en la lectura e interpretación 
Tabla 3 Análisis multivariable. Desenlace muerte

\begin{tabular}{llllllc}
\hline Variable & Coef & Std.E & $\mathrm{t}$ & $\mathrm{P}>|\mathrm{t}|$ & \multicolumn{2}{c}{ [Intervalo de confianza 95\%] } \\
\hline Edad & $-0,00015$ & 0,0010 & $-0,15$ & 0,88 & $-0,002$ & 0,0018 \\
Tiempo de ejercicio & $-0,00429$ & 0,0043 & $-0,99$ & 0,32 & $-0,012$ & 0,0042 \\
Capacidad de ejercicio & $-0,00173$ & 0,0045 & $-0,38$ & 0,70 & $-0,106$ & 0,0072 \\
Índice ST/FC & 0,02862 & 0,0198 & 1,44 & 0,15 & $-0,106$ & 0,0679 \\
Índice pendiente ST/FC & 0,0131 & 0,0149 & $-0,88$ & 0,37 & $-0,042$ & 0,0163 \\
Cons & 0,05332 & 0,0850 & 0,63 & 0,53 & $-0,114$ & 0,2214 \\
\hline
\end{tabular}

Cons: Constante.

Tabla 4 Análisis multivariable. Desenlaces cardiovasculares

\begin{tabular}{lllrrrr}
\hline Variable & Coef & Std.E & \multicolumn{1}{c}{$\mathrm{t}$} & $\mathrm{P}>|\mathrm{t}|$ & \multicolumn{2}{c}{ [Intervalo de confianza 95\%] } \\
\hline Edad & $-0,00004$ & 0,0023 & $-0,00$ & 1 & $-0,004$ & 0,004 \\
Tiempo de ejercicio & $-0,01705$ & 0,1026 & 1,66 & 0,09 & $-0,003$ & 0,73 \\
Capacidad de ejercicio & $-0,02362$ & 0,1072 & $-2,20$ & 0,029 & $-0,044$ & $-0,002$ \\
Índice ST/FC & 0,14586 & 0,0470 & 3,10 & 0,020 & 0,052 & 0,238 \\
Índice pendiente ST/FC & $-0,06709$ & 0,0353 & $-1,90$ & 0,06 & $-0,136$ & 0,027 \\
Cons & 0,05151 & 0,2014 & 0,26 & 0,79 & $-0,46$ & 0,450 \\
\hline
\end{tabular}

Cons: Constante.

Tabla 5 Análisis multivariable. Hospitalización

\begin{tabular}{llllllc}
\hline Variable & Coef & Std.E & $\mathrm{t}$ & $\mathrm{P}>|\mathrm{t}|$ & \multicolumn{2}{c}{ [Intervalo de confianza 95\%] } \\
\hline Edad & $-0,0032$ & 0,0038 & $-0,86$ & 0,39 & $-0,010$ & 0,004 \\
Tiempo de ejercicio & $-0,0054$ & 0,0160 & $-0,33$ & 0,74 & $-0,037$ & 0,73 \\
Capacidad de ejercicio & $-0,0063$ & 0,0170 & $-0,38$ & 0,70 & $-0,040$ & $-0,002$ \\
Índice ST/FC & 0,0146 & 0,0747 & $-0,20$ & 0,84 & $-0,162$ & 0,238 \\
Índice pendiente ST/FC & 0,0201 & 0,0561 & 0,36 & 0,72 & $-0,090$ & 0,027 \\
Cons & 0,4874 & 0,3202 & 1,52 & 0,13 & $-0,146$ & 1,120 \\
\hline
\end{tabular}

Cons: Constante.

de la prueba de esfuerzo convencional. En este trabajo, se realizó la evaluación de la capacidad de predicción de eventos cardiovasculares mediante la medición y uso de los índices pendiente ST/FC y $\Delta \mathrm{ST} / \Delta \mathrm{FC}$, obteniendo una discreta mejoría en la capacidad de predicción global y relación significativa desde el punto de vista estadístico del índice pendiente ST/FC con los desenlaces cardiovasculares descritos en el trabajo, que a su vez se relaciona con la capacidad del ejercicio desarrollada durante la prueba, variable que ya se conoce, tiene esta implicación. El pobre desempeño de estos índices también podría verse afectado por el número de eventos en la población en la cual se analizaron estas variables (solo dos muertes) y la buena capacidad del ejercicio promedio durante los estudios.

Como principales limitaciones de este trabajo se encuentra el sesgo de recuerdo y la medición de los desenlaces cardiovasculares, el cual se intentó atenuar mediante el cálculo del tamaño de muestra y análisis de sensibilidad descrito, así mismo, el tiempo de evaluación del desarrollo de los desenlaces podría ser precoz, si se compara con otros índices que miden el desarrollo de los eventos a 5 años, lo que se plantea como un objetivo para la segunda fase de este trabajo.

\section{Conclusiones}

El uso del índice pendiente ST/FC se encuentra relacionado estadísticamente con el desarrollo de desenlaces cardiovasculares un año luego de la realización de la prueba, logrando un discreto aumento de la capacidad de predicción global de la prueba de esfuerzo convencional (AUC 0,70 a AUC 0,74).

No se encontró relación estadísticamente significativa o mejoría de la capacidad de predicción global de eventos cardiovasculares con la utilización del índice $\Delta \mathrm{ST} / \Delta \mathrm{FC}$.

\section{Responsabilidades éticas}

Protección de personas y animales. Los autores declaran que los procedimientos seguidos se conformaron a las normas éticas del comité de experimentación humana responsable y de acuerdo con la Asociación Médica Mundial y la Declaración de Helsinki.

Confidencialidad de los datos. Los autores declaran que han seguido los protocolos de su centro de trabajo sobre la publicación de datos de los pacientes. 
Derecho a la privacidad y consentimiento informado. Los autores declaran que en este artículo no aparecen datos de los pacientes.

\section{Financiación}

\section{Ninguna.}

\section{Conflictos de intereses de los autores}

Los autores manifiestan no tener ningún conflicto de intereses.

\section{Bibliografía}

1. WHO. Global atlas on cardiovascular disease prevention and control. Geneva: World Health Organization, 2011.

2. Okin PM, Kligfield P. Gender-specific criteria and performance of the exercise electrocardiogram. Circulation. 1995;92:1209-16.

3. Gibbons RJ, Balady GJ, Beasley JW, FAAFP, JohnW, Bricker J, Timothy, et al. ACC/AHA Guidelines for Exercise Testing: Executive Summary. Circulation. 1997;96:345-54.

4. A Report of the American College of Cardiology/American Heart Association Task Force on Practice Guidelines (Committee on Exercise Testing). Circulation. 1997;96:45-354.
5. Blacklock SM. The symptom of chest pain in Family Practice. J Fam Pract. 1977;4:429-33.

6. Lampe FC, Whincup PH, Wannamethee SG, Ebrahim S, Walker M, Shaper AG. Chest pain on questionnaire and prediction of major ischaemic heart disease events in men. Eur Heart J. 1998;19:63-73.

7. Adabag AS, Grandits GA, Prineas RJ, Crow RS, Bloomfield HE, Neaton JD. Relation of heart rate parameters during exercise test to sudden death and all-cause mortality in asymptomatic men. Am J Cardiol. 2008;101:437-43.

8. Gianrossi R, Detrano R, Mulvihill D, Lehmann K, Dubach P, Colombo A, et al. Exercise-induced ST depression in the diagnosis of coronary artery disease. A meta-analysis. Circulation. 1989;80:87-98.

9. Mark DB, Hlatky MA, Harrell FE Jr, Lee KL, Califf RM, Pryor DB. Exercise treadmill score for predicting prognosis in coronary artery disease. Ann Intern Med. 1987;106:793-800.

10. Lauer MS, Pothier CE, Magid DJ, Smith SS, Kattan MW. An externally validated model for predicting Long-term survival after exercise treadmill testing in patients with suspected coronary artery disease and a normal electrocardiogram. Ann Intern Med. 2007; 147:821-8.

11. Kardash M, Elamin MS, Mary DA, Whitaker M, Smith DR, Boyle $\mathrm{R}$, et al. The slope of ST segment/heart rate relationship during exercise in the prediction of severity of coronary artery disease. Eur Heart J. 1982;3:449-58. 